\title{
GREEN TEETH ASSOCIATED WITH HIRSCHPRUNG'S DISEASE- A RARE CASE REPORT.
}

Swati Goyal, Piyush Mittal
1. Assistant Professor. Department of Pedodontics and Preventive Dentistry, Govt. Dental College and Hospital, Ahmedabad, Gujarat.
2. Assistant Professor. Department of Orthopaedics, B. J. Medical College and Hospital, Ahmedabad, Gujarat.

\section{CORRESPONDING AUTHOR:}

Dr. Swati Goyal, J-404, Shilalekh Society, Opposite Police stadium, Subash bridge, Shahibaug, Ahmedabad-380004, E-mail: geniusgoyal@yahoo.co.in, Ph: 00919909947117

ABSTRACT: Green pigmentation of primary teeth is an uncommon condition which is associated with the deposition of bilirubin in the dental hard tissues. Prolonged hyperbilirubinemia has long been recognized as a frequent clinical manifestation of infection in newborn, although the green staining of teeth caused by Hirschprung's disease has not yet been reported. The purpose of this report is to present a case of green teeth in primary dentition in a 17-month old Indian boy. Medical history revealed systemic problems during the neonatal period of an infectious, neurological, renal and digestive in nature along with a high amount of serum bilirubin levels. The green pigmentation of primary teeth was due to hyperbilirubinemia caused by these systemic conditions. The reported case confirms the relevance of past medical history in establishing the diagnosis and the etiology of green primary teeth. The presence of green pigmentation in teeth is an indication of early hyperbilirubinemia which can determine when in life this disorder occurred. Thus, even if there is no prior information about a patient with green teeth, it is possible to estimate the type of systemic disorder he/she had previously and how long he/she had the disease.

KEYWORDS: green teeth, hyperbilirubinemia, bilirubin, jaundice.

MESH TERMS: primary teeth, meconium, infant.

INTRODUCTION: Hyperbilirubinemia is chemically defined as a serum concentration of bilirubin greater than $1.5 \mathrm{mg} / 100 \mathrm{~mL}$. It can result from a variety of problems experienced in the neonatal period. Bilirubin is extensively deposited throughout the body during hyperbilirubinemia, although it disappears from the soft tissues after remission. ${ }^{1}$ Nevertheless, in hard tissues bilirubin is permanently trapped, because after maturation these tissues lose metabolic activity. ${ }^{2}$

Neonatal jaundice associated with elevated levels of serum bilirubin is never physiologic. It can be the result of infection, metabolic, genetic and anatomic abnormalities that can result in either mechanical obstruction of bile flow or impairment of the hepatic excretory function and bile secretion. Bacterial infection and septicemia is a frequent cause of an increase in conjugated bilirubin. Escherichia coli have been the most common organism reported.1, ${ }^{3}$. In the present case also the blood cultures were positive for E. coli species. This bacterial infection resulted in septicemia secondary to the Hirschprung's disease also known as Congenital 
Aganglionic Megacolon causing functional colonic obstruction resulting in severe constipation and abdominal distension. Considering the green pigmentation of teeth due to the Hirschprung's disease is an uncommon condition, the present case is reported.

CASE REPORT: A 17 month old Indian boy reported to the department with the chief complaint of green discoloration of teeth. A complete history and thorough intra-oral examination was done to evaluate this case. The patient was the first child who had full-term birth after an uncomplicated pregnancy. But, the child had congenital hydrocephalus; he was vomiting after every feed and had developed abdominal distension due to non-passage of stools since 3-4 days after birth (failure to pass meconium in first $24 \mathrm{hrs}$ after birth).The abdominal sonography was done which showed multiple dilated bowel loops and enlarged colon. The renal function test showed increase in urea and creatinine levels. The total serum bilirubin level $5.3 \mathrm{mg} / \mathrm{dl}$ and direct reacting bilirubin $0.1 \mathrm{mg} / \mathrm{dl}$ was present which were progressively increasing to $18.3 \mathrm{mg} / \mathrm{dl} \& 7.3 \mathrm{mg} / \mathrm{dl}$ respectively after 6 days and highest $30.3 \mathrm{mg} / \mathrm{dl}$ of total serum bilirubin and $26.2 \mathrm{mg} / \mathrm{dl}$ direct bilirubin was noted after 10 days.

The child was diagnosed with Hirschprung's disease on the basis of symptoms and the abdominal sonography report. The disease is caused by the failure of ganglion cells to migrate cephalocaudally through the neural crest during weeks four to 12 of gestation, causing an absence of ganglion cells in all or part of the colon. ${ }^{4}$ Varying lengths of the distal colon are unable to relax, causing functional colonic obstruction over time.

He developed E.coli infection and sepsis due to the colonic obstruction and in turn hyperbilirubinemia. The child was medicated and underwent corrective surgery of colon. The patient improved gradually and was discharged when the laboratory and sonography tests were normal. The patient was under follow-up and was continuous monitored; the parents were also counseled and instructed for high-fiber diet for the child in future. The intra-oral examination when the child reported at 17 months showed green intrinsic staining of all the erupted primary teeth (chlorodontia). There were no mucosal or gingival abnormalities and all teeth were noncarious. The green pigmentation was present from middle $3^{\text {rd }}$ to cervical $3^{\text {rd }}$ while the incisal and occlusal areas showed a tinge of discoloration (Fig 1).

The child also had congenital deafness with left ear and partial with right ear. The child is now being treated for the same with a speech therapist.

DISCUSSION: There is no estimate, but the prevalence of green teeth is low. The literature provides data for 48 cases. Green stained teeth have been reported in infants and children with an age range of newborn to 11 years. The sex was reported in 39 cases; 20 were girls $(51.3 \%)$ and 19 were boys (48.7\%). These findings suggest a lack of gender susceptibility. ${ }^{5}$

The cause of green teeth was determined through the medical history in 43 cases: 58.1 $\%$ ( 25) was caused by biliary atresia, 30.2\%(13) by hemolytic disease, $2.3 \%$ (1) by absence of bile ducts, $2.3 \%$ (1) by hemolysis in utero, $2.3 \%$ (1) by biliary hypoplasia, and $2.3 \%$ (1) by cholestasis associated with sepsis. ${ }^{5}$ Green teeth associated with Hirschprung's disease has not yet been reported.

Hirschprung's disease (HSCR), first described by Harald Hirschsprung in 1888 is the main genetic cause of functional intestinal obstruction with an incidence of 1/5000 live births. The cause of Hirschsprung's disease is multifactorial, and the disease can be familial or develops spontaneously. Hirschsprung's disease (congenital megacolon) is caused by the failed migration of colonic ganglion cells during gestation. The diagnosis is confirmed with a rectal suction 
biopsy, which shows the absence of ganglion cells and the presence of hypertrophic nerve trunks. ${ }^{6}$ This developmental disorder is a neurocristopathy (disease arising from maldevelopment of neural crest) and is characterized by the absence of the enteric ganglia along a variable length of the intestine. The ganglion cells are derived from the "neural crest" of the embryo. Any failures of these cells to grow, survive, migrate, or mature in the embryonic gut, could result in Hirschsprung disease (HSCR). Hirschsprung disease is caused by genetic changes (mutations) in the DNA sequence. The genetic changes that lead to Hirschsprung disease can come from several sources. Some individuals may have a new change, one that is not present in either parent, but arose at the time of conception. Other individuals may have inherited, from their parents, a particular combination of subtle genetic changes that gave rise to Hirschsprung disease. The parents may or may not be affected. In the majority of families, Hirschsprung disease occurs as an isolated case, meaning there is no family history of the disease. So far, eight genes have been found to be involved in HSCR. This frequent congenital malformation now stands as a model for genetic disorders with complex patterns of inheritance. HSCR occurs as an isolated trait in $70 \%$ of cases. A chromosomal abnormality is associated with it in $12 \%$ of cases, trisomy 21 being by far the most frequent (>90\%). ${ }^{7}$ Varying lengths of the distal colon are unable to relax, causing functional colonic obstruction. Symptoms range from neonatal intestinal obstruction to chronic progressive constipation in older children. It is more common in boys than girls. Associated congenital anomalies are found in $18 \%$ of HSCR patients. Hirschsprung's disease is associated with neurologic, cardiovascular, urologic, and gastrointestinal abnormalities. Conditions that have been linked to Hirschsprung's disease include congenital deafness, hydrocephalus, diverticulum of the bladder, Meckel's diverticulum, imperforate anus, ventricular septal defect, renal agenesis. ${ }^{8}$

Approximately 80 percent of patients present in the first few months of life with difficult bowel movements, poor feeding, and progressive abdominal distention. Up to 90 percent of infants with Hirschsprung's disease fail to pass meconium in the first 24 hours of life and become prone for infection.

Jaundice has long been recognized as a clinical manifestation of infection in the newborn and during infancy. The endotoxin of E.coli has been reported to cause cholestasis (defect in excretion of biliary salts) by inhibiting the bile-salt -independent fraction (BSIF). ${ }^{9}$

It is seen that there is variation in the intensity of green staining which can be correlated with the duration and depth of jaundice. ${ }^{10}$ Miller, ${ }^{11}$ considered that to provoke obvious green staining of teeth, the bilirubin concentration must exceed $30 \mathrm{mg} / 100 \mathrm{~mL}$. When the microscopic analysis of green teeth was done; first reported by Lorsch et al, 12 it was seen that there were bright green neonatal lines in enamel and dentin with a tendency to fracture.

It is seen that the primary teeth were the most frequently affected. The green pigmentation on the teeth occurred during the dental tissue formation because of the systemic problems \& in turn hyperbilirubinemia during the neonatal period. The deciduous tooth calcification process begins in the $4^{\text {th }}$ month of intrauterine life finishing 11 months after birth. Primary teeth in formative stage are affected by the excess bilirubin \& become intrinsically stained. There is no evidence as to how the pigment is incorporated; it can probably diffuse through dentin which is highly permeable. This green pigment was suggested to be biliverdin which has now recently been shown to be bilirubin. ${ }^{13}$

The diagnosis of green teeth is usually based on the medical history and duration of jaundice and hyperbilirubinemia in these patients. The differential diagnosis of green teeth includes extrinsic discolorations caused by chromogenic bacteria or medicaments and intrinsic 
discolorations caused by dentinogenesis imperfect, amelogenesis imperfecta, tetracycline and congenital erythropoietic porphyria.

Managing parental anxiety demands from the clinician accurate predictions about possible damage to the unerupted permanent dentition. Knowledge of the chronologic development of teeth is essential, in conjugation of the history of total bilirubin levels, to predict the degree and pattern of green discoloration that will be found in the permanent dentition.

Cosmetic treatment of discolored deciduous teeth becomes increasingly important as the child grows older. This will assist social interaction and stimulate greater self-esteem in the child.

Treatment considerations for green-stained teeth include restorative composite crowns; ceramic crowns resin restorations and bleaching techniques. The approach suggested by Giunta and Tsamtsouris ${ }^{14}$ involves the use of transillumination with the ultraviolet light to accelerate the breakdown and dissipation of bilirubin products. It will be interesting to investigate whether pigmentation of the cusps of the first permanent molar occurs in this case because green staining appeared to be present only in those dental tissues formed after birth and to analyze exfoliated teeth microscopically.

ACKNOWLEDGMENTS: The author is thankful to the KLE Hospital (Belgaum, Karnataka) management and staff for furnishing the detailed medical records of the patient.

\section{REFERENCES:}

1. Schiff L. Schiff E R. Diseases of liver. Philadelphia: J B Lippincott; 1993.p.1099-203.

2. Wantabe K, Shibata T, Kurosawa T, Morisaki I, Kinehara M, Igarashi S, et al. Bilirubin pigmentation of human teeth caused by hyperbilirubinemia. J Oral Pathol Med 1999; 28:128-30.

3. Bernstein J, Brown AK. Sepsis and jaundice in early infancy. Pediatrics 1962; 29:873-82.

4. Parisi MA, Kapur RP. Genetics of Hirschprung disease. Curr Opin Pediatr 2000; 12:610-7.

5. Luciana PG, Tarcilia AS. Green teeth associated with cholestasis caused by sepsis: A case report and review of literature. Oral Surg Oral Med Oral Pathol Oral Radiol Endod 2003; 95:446-51.

6. De Lorijin F, Reistsma JB, Voskuijl WP, Aronson DC, Ten Kate FJ, Smets AM, et al. diagnosis of Hirschprung's disease: a prospective, comparative accuracy study of common tests. J Pediatr 2005; 146:787-92.

7. Amiel J, Lyonnet S. Hirschprung disease, associated syndromes, and genetics: a review. J Med Genet 2001; 38: 729-39.

8. Stewart DR, Von Allmen D. The genetics of Hirschprung disease. Gastroenterol Clin North Am 2003; 32:819-37.

9. Utili R, Abernathy CO, Zimmerman HJ. Inhibition of $\mathrm{Na}+, \mathrm{K}+$ adenosinetriphosphatase by endotoxin- induced cholestasis. J Infect Dis 1977; 136:583-7.

10. Tank G. Two cases of green pigmentation of the deciduous teeth associated with hemolytic disease of newborn. JADA 1951; 42:302-6.

11. Miller J. The effect of bilirubin on tooth development in the rat. Arch Oral Biol 1960; 2:143-50.

12. Lorsch PK, Brown JC, Boyle PE. Staining of the dental structure in jaundice of the newborn. J Dent Res 1940; 19:293. 
13. Shibata T, Wantabe K, Oda H, Arisue M, Kurosawa T, Toma M, et al. Experimental bilirubin pigmentation of rat dentine and its detection by a quantitative analytical method. Arch Oral Biol 1996; 41:509-11.

14. Giunta J, Tsamtsouris A. Stains and discolorations of teeth: review and case reports. J Pedod 1978; 2:175-82.

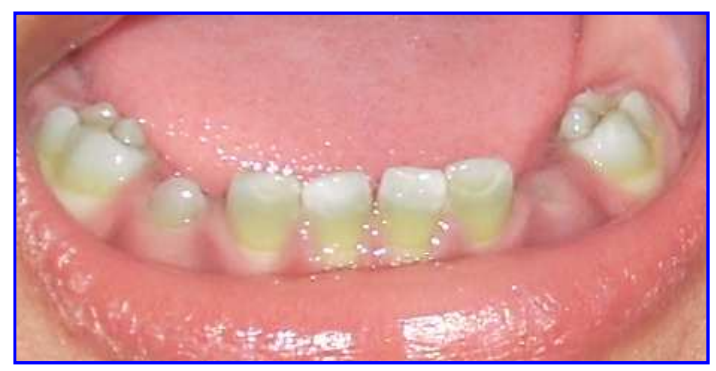

Fig 1: Clinical appearance of the lower primary teeth at 17 month of age, demonstrating green pigmentation 